\title{
High-elevation synoptic radio array for detection of upward moving air-showers, deployed in the Antarctic mountains
}

\author{
Jiwoo Nam*, \\ National Taiwan University, Taipei, Taiwan \\ 1 Roosevelt Road, Taipei, Taiwan \\ E-mail: jwnam@phys.ntu.edu.tw
}

(TAROGE Collaboration)

Pisin Chen ${ }^{1}$, Y.C. Chen ${ }^{1}$, S.-Y. Hsu' ${ }^{1}$, J.-J. Huang ${ }^{1}$, M.-H. A. Huang ${ }^{2}$, C.-Y. Kuo ${ }^{1}$, C.H. Leung $^{1}$, T.C. Liu ${ }^{1}$, B.K. Shin ${ }^{1}$, Y.-S. Shiao ${ }^{1}$, M.-Z. Wang ${ }^{1}$, S.-H. Wang ${ }^{1}$, Y.-H. Wang ${ }^{1}$

${ }^{1}$ Dept. of Physics, Grad. Inst. of Astrophys., \& Leung Center for Cosmology and Particle Astrophysics, National Taiwan University, Taipei, Taiwan

${ }^{2}$ Department of Energy and Resources, National United University, Miao-Li, Taiwan

\section{Z. Besson ${ }^{3,4}$, C. Hornhuber ${ }^{3}$, A. Novikov ${ }^{3,4}$}

${ }^{3}$ Dept. of Physics and Astronomy and Instrumentation Design Laboratory, University of Kansas, Lawrence, KS, USA

${ }^{4}$ National Research Nuclear University, Moscow Engineering Physics Institute, Moscow, Russia

\section{and the ARIANNA Collaboration}

\footnotetext{
We present a new radio array experiment on the top of the highest mountains in Antarctica, to detect upward moving air-showers similar to those recently reported by the ANITA experiment. The detection concept follows ANITA, but has significantly greater livetime and extendibility. We propose to install 10 stations over the next 5 years to obtain sufficient sensitivity to test ANITA's 'mystery events'. As the first step, we installed a prototype station (TAROGE-M) consisting of 5 LPDA antennas, atop Mt. Melbourne, Antarctica at an elevation of $2730 \mathrm{~m}$, in FebMar 2019. We present the scientific potential, detector design and construction, as well as the initial performance of the prototype station.
}

36th International Cosmic Ray Conference -ICRC2019-

July 24th - August 1st, 2019

Madison, WI, U.S.A. 
The ANtarctic Impulsive Transient Antenna (ANITA) collaboration recently reported the observation of two anomalous upward-moving air-shower events with energies of $\sim 0.6 \times$ $10^{18} \mathrm{eV}$, observed during the first and the third flights [1,2]. One may interpret them as decays of $\tau$ leptons in the atmosphere emerging from the Antarctic surface after CC interactions of $v_{\tau}$ under the surface. However, several thousand kilometers of neutrino propagation lengths through earth, which correspond to the observed elevation angles of those events, $-27.4^{\circ}$ and $-35.0^{\circ}$ respectively, are too long to be explained by the standard model (SM). There are many of theoretical proposals to explain these events in beyond standard model [3-8]. An alternative explanation these events to be cosmic ray signals, but reflected on subsurface layers and firn density inversions has been proposed [9]. It is important to obtain more numbers of events in order to reveal the origin of these events. However, rather than ANITA, there is no other experiment going on or being planned at this moment.

We propose a new radio array experiment on the top of high mountains in Antarctica, which is utilized to detect upward moving air-showers. We follow the ANITA's experimental concept; detecting ultra-wide band impulsive radio frequency (RF) signals emitted by the air-shower as a result of its interaction with the geomagnetic field, using multiple RF receivers, and applying the interferometric technique for event reconstruction [10]. On the other hand, our approach is to locate antennas on the top of mountains at $\sim 3 \mathrm{~km}$ altitude instead of using balloon at much higher altitude $\sim 35 \mathrm{~km}$. While ANITA obtains a huge target volume in high energies above $10^{19} \mathrm{eV}$ because of its high altitude, our method is able to lower the energy threshold by placing the antennas closer to the showers than the balloon-borne experiment. Furthermore, this method enhances the effective volume due to dense rock target in nearby mountains while ANITA uses ice as a target for neutrino interactions. The most important advantages of our approach is in livetime. Comparing to the fact that the typical flight duration of the NASA long duration balloons, which ANITA relies on, is limited to be about 30 days, our detector located on the ground basically can operate at least half a year during Austral summer using a solar power system. The operation of remote antenna stations for six months in the Antarctic environment has already been proven experimentally using a solar power system [11]. Furthermore, winter operation is highly possible using wind turbine system, which can double the exposure. The extendibility is another great advantage in our approach. With the help of easy-to-install RF devices, it is quite possible to install multiple stations in a relatively short time.

Antarctic mountains are ideal for this experiment due to the quietest ambient noise and the strongest geo-magnetic field. We found Mt. Melbourne to be highly attractive places satisfying following conditions; 1) a high elevation as $2.7 \mathrm{~km}, 2$ ) a clear target view of high mountain ridges within the horizon, 3) no anthropogenic RF noise sources (stations or camps) in the field of view, 4) a good transportation method by helicopter, 5) a wide and snow-free available for installation of instruments around the top, and 6) scientific supports and infrastructure available at Jangbogo station, Korea Polar Research Institute, etc. Figure 1 shows a satellite map near Mt. Melbourne. The Jangbogo station which locates at $\sim 33 \mathrm{~km}$ way from the site can be possible sources of anthropogenic RF noise. However, facing receiver antennas toward the other direction minimizes the effect. 


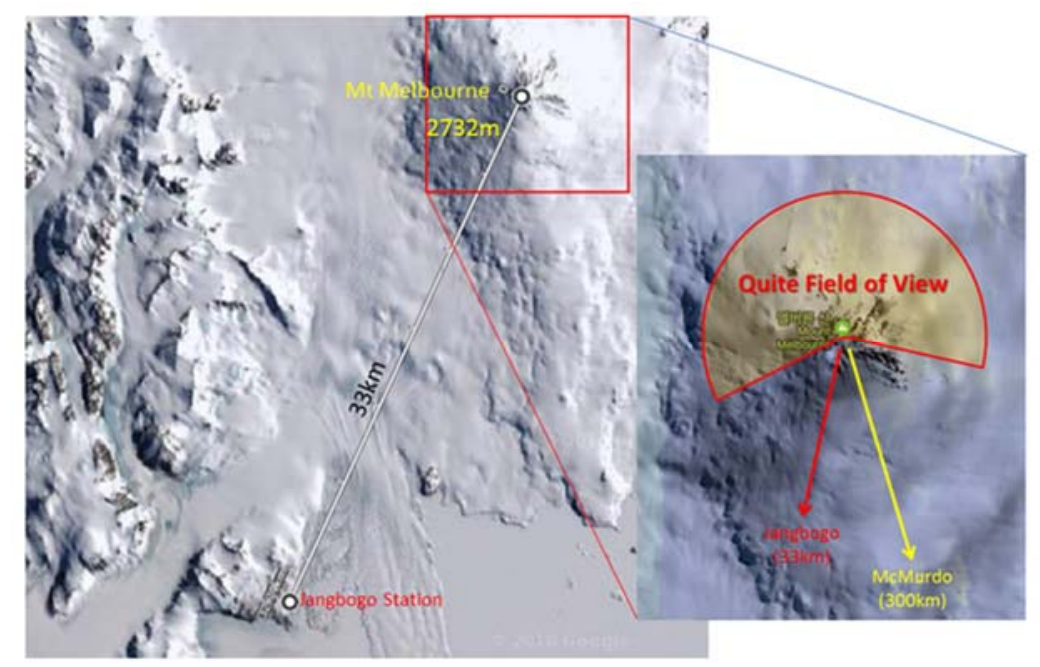

Figure 1: Satellite map near Mt. Melbourne

The design of our instrument is basically inherited from the heritage from existing projects; the ARIANNA-HCR [11] and TAROGE experiment [12], which have demonstrated its detection capability and robust operations in the harsh environment in Taiwan's high mountain as well as Antarctica for several years. A station consists of 8 LPDA antennas; 4 horizontal polarization (Hpol) channels and 4 vertical polarization (V-pol) channels. This dual polarization configuration is to measure polarization angles of observed signals, which allows a confirmation if the signals are induced at the air-shower interaction with the geo-magnetic field. The multiple antennas are used to measure arrival direction of the signal using the interferometry technique. The left panel of Figure 2 shows the design of an antenna tower. The station has 4 towers forming a triangular antenna array as shown in the right panel of Figure 2. The pointing resolution on the arrival signal is crucial to distinguish up-coming air-shower signals from down-going cosmic ray showers in near horizon. The required pointing resolution, $<0.2$ degrees, is achievable when the antenna array has vertical baselines longer than 4 meters with high speed $2 \mathrm{GHz}$ digitizer. The triangular tower array installed on the mountain slope is a practically easiest solution to obtain the required vertical baselines. We use Log-periodic dipole array (LPDA) antennas as receivers. We designed LPDA for a gain $\sim 7 \mathrm{dBi}$ in a frequency range $150-500 \mathrm{MHz}$ which corresponds to a half-power beam width (HPBW) $\sim 60$ degrees. The LPDA has an excellent endurance for strong winds, therefore, is a suitable design for applications in the Antarctic high mountain environment as demonstrated in TAROGE and ARIANNA-HCR experiments. Solar panels (Photovoltaic) panels and a vertical wind-turbine are mounted on the side of the towers. 

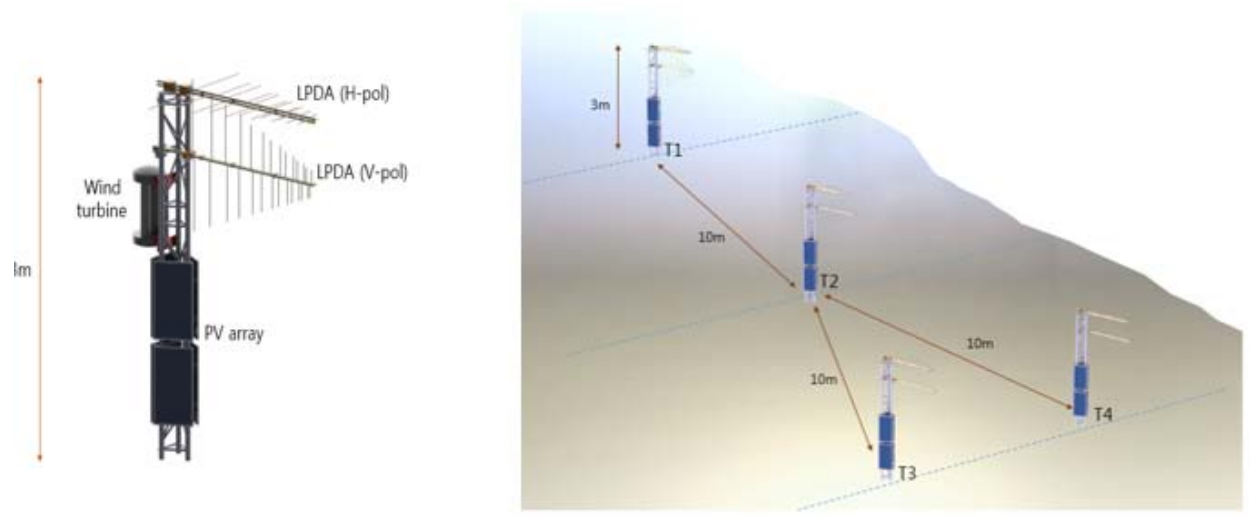

Figure 2: Receiver tower assembly (left) and tower allocation (right).

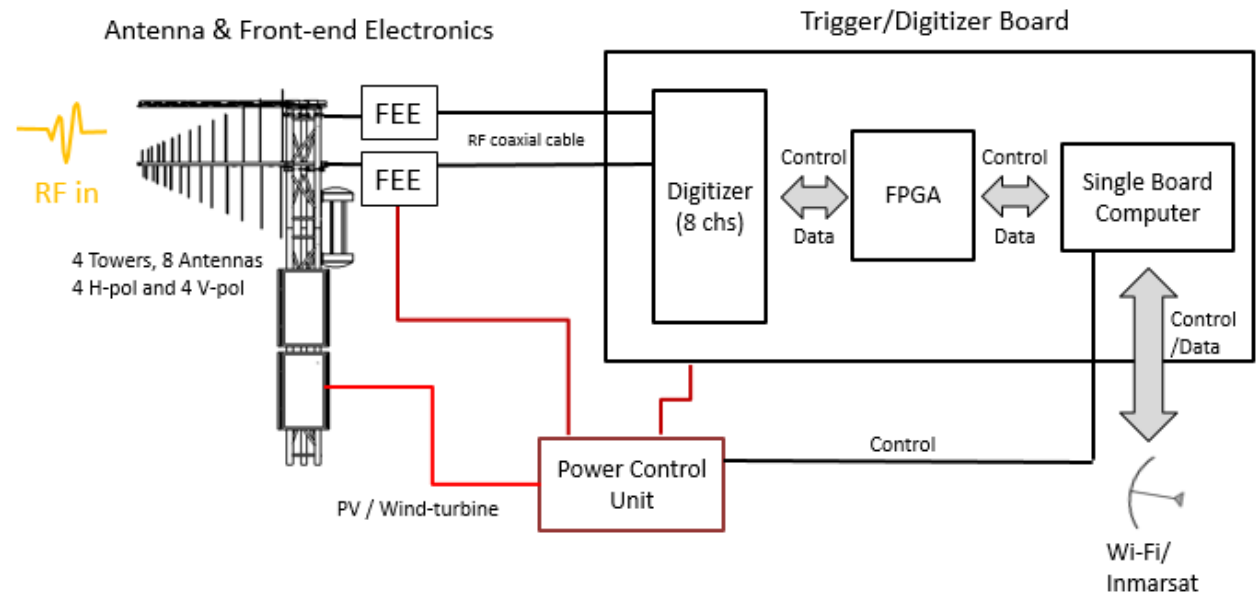

Figure3: The system diagram.

Figure 3 shows the system diagram of the station. Received signal in the antenna is fed into the trigger/digitizer units through the RF front-end unit. The front-end unit consists of filters and Low Noise Amplifiers (LNAs). The required system gain is $65 \mathrm{~dB}$ which is obtained by combining a LNA (+35dB) and a second stage amplifier $(+30 \mathrm{~dB})$. The trigger/digitizer board which has been developed by ARIANNA collaboration, is utilized for radio neutrino observatory for Antarctic environment; extremely low temperature, low power consumption, a powerful trigger capability. The unit has total 8 channel readout with two of 4 channel Synchronous Sampling and Trigger (SST) chips. It reads-out individually with a sampling rate of $2 \mathrm{GHz}$, providing a bandwidth of up to $1 \mathrm{GHz}$. Once a trigger given, waveforms of 256 samples are digitized with 12 bit ADCs and read-out in FPGA. The trigger uses the double-threshold technique in time domain. It requires a coincidence of positive- and negative-voltage threshold crossings to be happened in a channel within 5 ns. Then a multi-channel coincidence, typically 3 out of 8 channels, is further applied. With a typical voltage threshold which is 4 times of rms voltage, the trigger can low event rates as low as $\sim 20 \mathrm{mHz}$. We further apply an online event filtering; rejection of continuous waveform (CW) events based on FFT and rejection of thermal events based on using the cross-correlation technique, can reduce the final event rate as low as $\mathrm{mHz}$. The data is stored locally and is also transmitted either via long-range WiFi or satellite to data servers in Northern hemisphere. The 
total power consumption for the station is less than 20W, thanks to low powered LNAs and the trigger/digitizer board. A couple of 30W PV panels would be good enough for typical summer operation, but 6-8 panels will be used to increase operation time spring and fall seasons. A hybrid power system (solar and wind) will be investigated to evaluate feasibility of the winter operation.

As the first step, a campaign to install a prototype station on the top of Mt. Melbourne was carried out during February-March 2019. The goals of the this campaign were following; 1) geographic survey, 2) Ambient RF survey, 3) validation of tower / antenna structures, 4) evaluation of accessibility and transportation by helicopter, 5) weather data acquisition, 6) test of wind turbine 7) test of satellite communication, and so on. We found a couple of suitable locations to install initial stations around the top with an excellent accessibility by helicopter. Several possible locations in $2300-2700 \mathrm{~m}$ were identified for the future extension. We installed

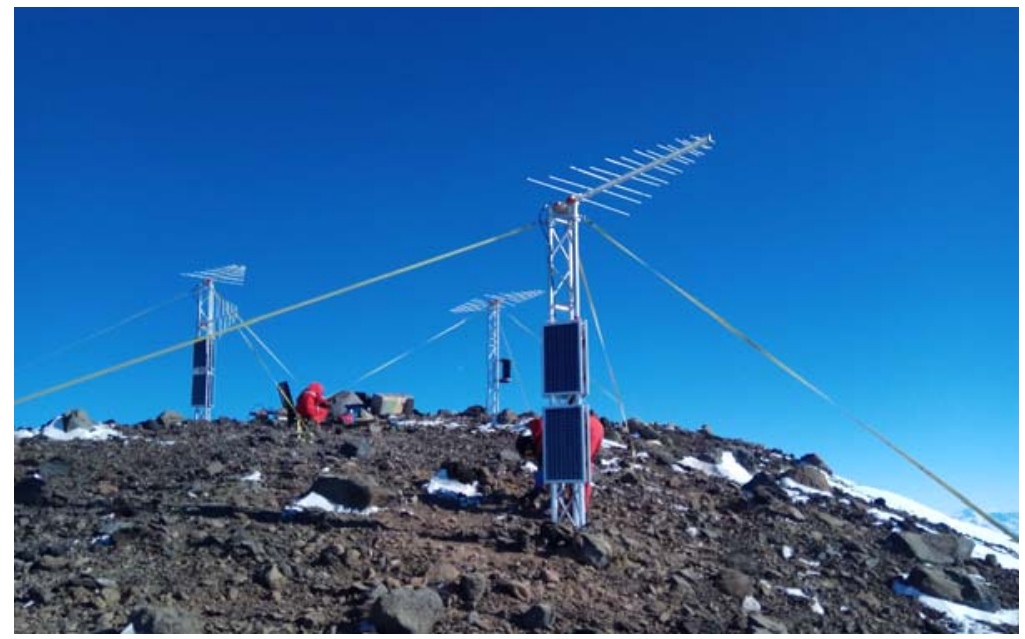

Figure 4: The prototype station installed on the top of Mt. Melbourne (March 2019)

the prototype station consisting of 5 LPDA antennas in 3 towers on the Northern slope around the top as seen in Figure 4. The installation went smoothly, and took about a total 48 men-hours on the site, which required 3 times of helicopter transportations for 3-4 passengers and 1 time for the instruments. The system operated successfully about 8 hours after installation completed, but lost the satellite communication afterward. As we faced the end of the summer operation schedule of Jangbogo station, following visits was not accomplished unfortunately, but a detailed investigation in future will identify the reason. Although only limited data were acquired, we obtained meaningful RF survey data which is important to determine future system design. Figure 5 shows average spectra of minimum-biased RF noise data obtained by the forced trigger at every 100 seconds. Except two CW peaks at $140 \mathrm{MHz}$ and $360 \mathrm{MHz}$, noise level is found to be quite low as similar at ARIANNA site in Moore's bay [11]. The CWs can be removed later by apply filters in future instruments. The small bump at the band of $240-280 \mathrm{MHz}$ was identified as satellite noise, but the effect to the sensitivity would be minimal because of its low strength. No impulsive noise event was observed during the 8 hours operation. Instead, we several events with modulated CW waveforms were recorded as displayed in on Figure 6. The CW filters to be installed in future will effectively suppress these events. 


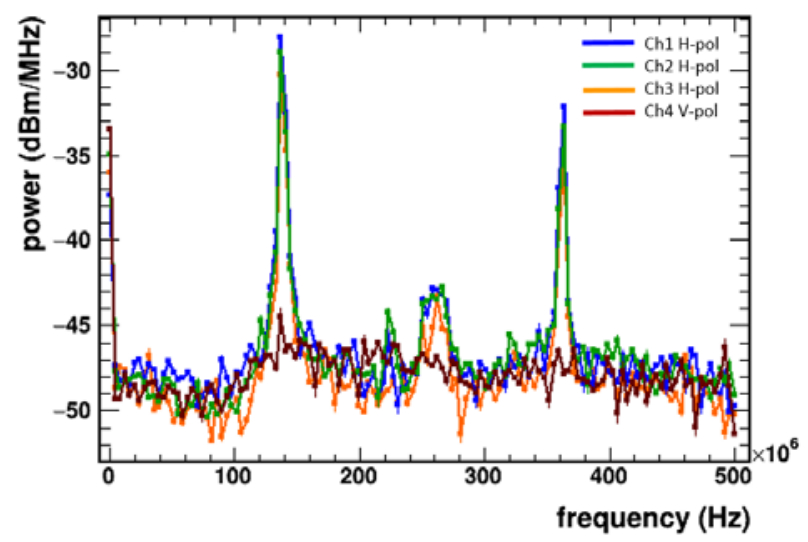

Figure 5: Ambient noise spectra obtained at Mt. Melbourne site.
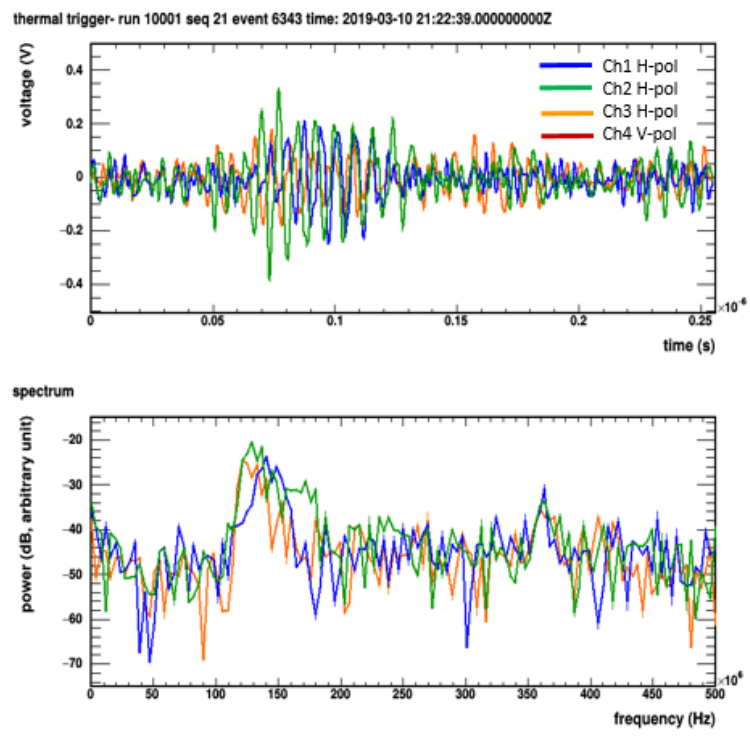

Figure 6: A sample of triggered noise events; time domain waveform (top) and FFT spectra (bottom)

We estimate detection capability of tau neutrinos by Monte-Carlo simulation. A software package; Simulation of High-energy Neutrinos Interaction with the Earth (SHINIE) [14] was used for neutrino interaction and tau propagation. CoREAS was used for modeling of radio emission from air-showers. In order to avoid a long CPU time required for simulation of very high energies in CoREAS simulation, we implemented a fast simulation method which parameterizes the strengths, spectra, and beam patterns of the radio emissions at lower energy $10^{16} \mathrm{eV}$, and scales them according to energies of showers and distances between air-shower and detector. This fast method provides a reasonable agreement of strength of E-field with the full CoREAS simulation within $20 \%$. The simulation takes into account the system response such as the LPDA antenna, the front-end amplifiers and filters. The same trigger algorithm and voltage threshold as ARIANNA-HCR were applied [11,13]. A realistic model of terrain around Mt. Melbourne was taken into account. 
Figure 7 shows results of the simulation. The top panel of the figure shows an estimated acceptance of the tau neutrino detection with a single station on Mt. Melbourne (red line) and the bottom panel shows estimations of exposures for four different scenarios on number of stations and duty cycles. The estimations for ANITA [17] are shown in black lines in both figures. As expected, our acceptance of the single station is two order of magnitude lower than ANITA's, mainly due to a smaller azimuth angle coverage and target volume in the field of view. However, the exposures taken into account operation time show promising capability of our approach. Even three stations which can be installed in a season practically, can obtain comparable exposures as ANITA in energies around $0.6 \times 10^{18} \mathrm{eV}$ in which the two anomalous events found. Assuming the exposure for tau neutrino detection to be similar as the anomalous event, our proposal with 10 stations would provide a sufficient exposure to test the anomalous events.
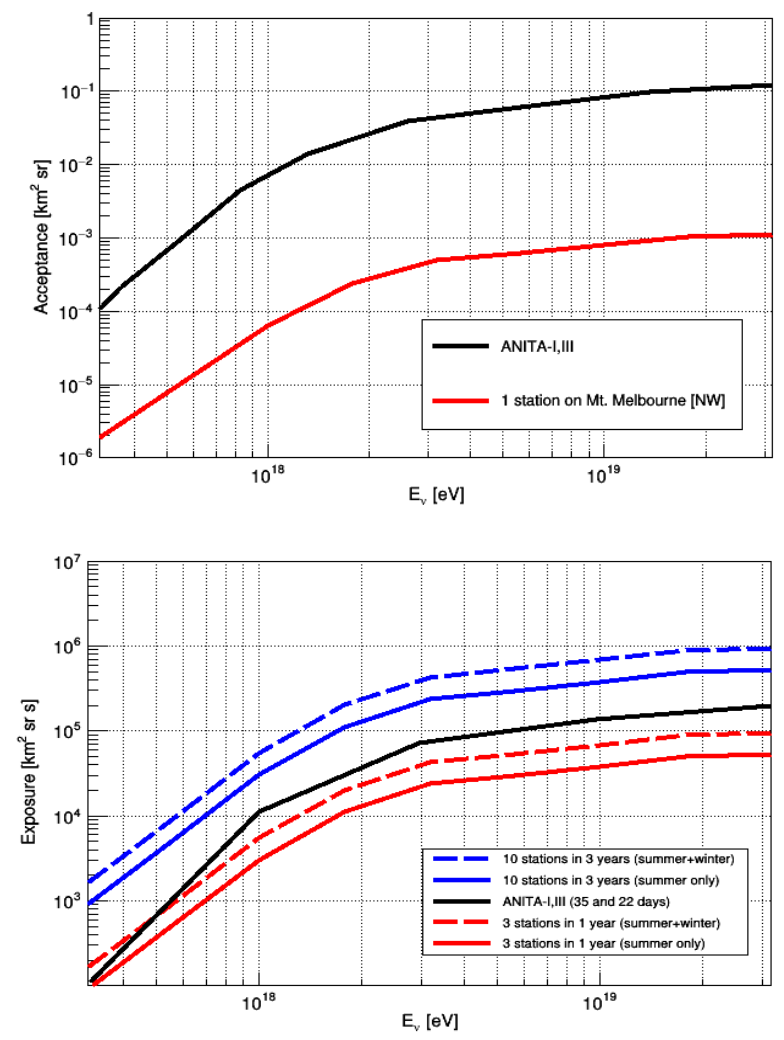

Figure 7: Simulation results on acceptance of the tau neutrino detection (top) and exposures (bottom).

We plan to upgrade the prototype station to a full-scale station adding more number of the receiver antennas in November-December 2019. Extension to build up-to 10 stations would be carried out in a couple of years, aiming to unveil the mystery of ANITA's upward-moving shower events.

We are thankful for support from Taiwan Ministry of Science and Technology, and Korea Polar Research Institute. We would like to extend our thanks to 2018-2019 on-ice Jangbogo station crews for their support. 


\section{References}

[1] P. W. Gorham et al. (ANITA Collaboration), Phys. Rev. Lett. 117, 071101, (2016).

[2] P. W. Gorham et al (ANITA Collaboration), Phys. Rev. Lett. 121, 161102 (2018).

[3] John F. Cherry and Ian M. Shoemaker, Phys. Rev. D 99, 063016 (2019).

[4] Gou-yuan Huang, Phys. Rev. D 98, 043019 (2018).

[5] Bhavesh Chauhan, Subhendra Mohanty, Phys. Rev. D 99, 095018 (2019)

[6] Luis A. Anchordoqui, Vernon Barger, John G. Learned, Danny Marfatia, Thomas J. Weiler, LHEP 1, $13(2018)$

[7] Emilian Dudas, Tony Gherghetta, Kunio Kaneta, Yann Mambrini, Keith A. Olive, Phys. Rev. D 98, 015030 (2018)

[8] Jack H. Collins, P. S. Bhupal Dev, Yicong Sui, Phys. Rev. D 99, 043009 (2019)

[9] Ian M. Shoemaker, Alexander Kusenko, Peter Kuipers Munneke, Andrew Romero-Wolf, Dustin M. Schroeder, Martin J. Siegert, arXiv:1905.02846

[10] A. Romero-Wolf et al. (ANITA Collaboration), Phys. Rev. D 99, 063011 (2019).

[11] S. H. Wang et al. (TAROGE \& ARIANNA Collaboration), Pos (ICRC 2017) 358 (2017)

[12] Jiwoo Nam et al. (TAROGE Collaboration), IJMPD, Vol25, No. 141645103 (2016)

[13] S. H. Wang et al. (TAROGE \& ARIANNA Collaboration), Pos (ICRC 2019) 462 (2019)

[14] M.A. Huang et al. Nucl. Phys. B (proc. supl.) Vol. 175-176, 472-475

[15] T. Huege, M. Ludwig, and C. W. James, arXiv:1301.2132

[16] Liu, H., K. Jezek, B. Li, and Z. Zhao. 2001. Radarsat Antarctic Mapping Project Digital Elevation Model, Version 2. 400m RAMP DEM data set. Boulder, Colorado USA. NASA National Snow and Ice Data Center Distributed Active Archive Center. [Date accessed in Dec 2016].

[17] A. Romero-Wolf et al. (ANITA Collaboration), Astroparticle Phys. 60, 72-85 (2015). 